\title{
Measuring Muscle Movements for Human Interfaces using a Flexible Piezoelectric Thin Film Sensor
}

\author{
Nan Bu, Junpei Tsukamoto, Naohiro Ueno, Keisuke Shima and Toshio Tsuji
}

\begin{abstract}
This paper proposes a novel method to measure muscle movements for human interfaces. During muscle movements, cross-sectional muscle area changes, and this can be detected at the skin surface. In this study, a flexible piezoelectric thin film sensor is used to measure the morphological change of the skin surface. This sensor is made of oriented aluminum nitride (AIN) thin film, and the total thickness is less than 40 $\mu \mathrm{m}$. Since the AlN film sensor has good sensitivity, small strain of the skin surface can be measured. Furthermore, a motion classification method is developed to investigate the potential of the proposed sensor for its use in human interfaces. Response characteristics of the AIN sensor were tested with experiments using a cantilever beam. In addition, motion classification experiments were conducted with five subjects, including a patient with cervical spine injury. The experimental results validate the effectiveness of the proposed method.
\end{abstract}

\section{INTRODUCTION}

Recording of muscle activity can be used to generate control signals for human-assisting manipulators and prosthetic devices. Since electromyography (EMG) signals, measured at the skin surface, can provide information about neuromuscular activities, surface EMG has been extensively used in a number of researches to detect human movement or intention of movement with a pattern classification process [1]-[3]. Up to the present, many EMG-based human interface applications have been proposed [4]-[6]. It has been suggested, however, that the amplitude of EMG is very low, and signals measured are usually affected by artifacts and noises. Many conscious efforts are required to achieve reliable control signals for human interfaces. Long-term usage may cause fatigue or stress in muscles. Moreover, electrodes are needed for sensing EMG signals. This also suffers from several drawbacks, including the fact that the conductive cream or gel is messy and often makes users feel uncomfortable, and changes of the skin-electrode impedance may affect signalto-noise ratio.

During contraction/relaxation, a muscle's cross-sectional area increases/decreases. This morphological change can be used to detect functional and contractile states of muscles. Several alternative methods have been investigated to measure change in muscle section dimension as a control signal [7]-[11]. Kenney et al. reported on measuring dimensional change in muscles using a transducer based on Hall effect

N. Bu and N. Ueno are with the Measurement Solution Research Center, National Institute of Advanced Industrial Science and Technology (AIST), 807-1, Shuku-machi, Tosu, 841-0052 JAPAN. bu@ieee.org

J. Tsukamoto, K. Shima and T. Tsuji are with the Department of Artificial Complex System Engineering, Hiroshima University, 1-4-1, Kagamiyama, Higashi-Hiroshima, 739-8527 JAPAN.

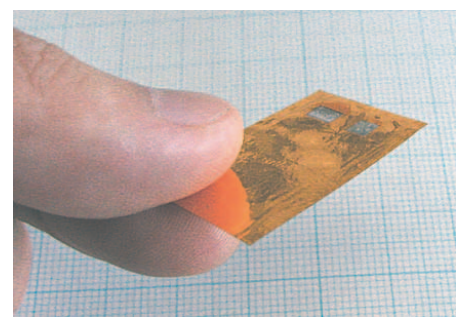

Fig. 1. A flexible AlN thin film sensor.

[7]. However, this transducer is bulky and inflexible, making the sensor uncomfortable and undesirably conspicuous, especially when used to the facial muscles.

The mechanomyography (MMG) signal, which reflects the mechanical vibration of skin surface, is also being explored. In [8], an accelerometer is used to acquire MMG signals, and the muscle belly radial displacement is obtained by integrating twice the MMG signals. On the other hand, root mean square features of MMG signals have been extracted for prosthetic control [9], [10]. When dealing with MMG signals, one must be careful and aware of possible large errors inherent, such as errors due to body/limb movements, noise of the accelerometer and signal processing circuits, and so on.

Furthermore, ultrasound imaging has been used for detecting muscle deformation during actions [11]. Since ultrasound imaging can trace muscle surfaces at different depths and different locations, this method is able to detect movements of individual muscles. However, ultrasound devices are quite expensive and large in size, it is not practical for human interface applications at the present.

This paper proposes a novel method to measure muscle movements as control signals for human interfaces. A flexible piezoelectric thin film sensor (see Fig. 1) is utilized to detect morphological changes of muscles at the skin surface. This sensor, proposed by Ueno et al. [12], is made of oriented aluminum nitride (AIN). The AlN layer is deposited on a flexible polyimide film, and the total thickness of this sensor is less than $40 \mu \mathrm{m}$. Flexibility makes this sensor fit well to the skin surface, and easy to be installed into supports like wristband. Unlike the methods proposed in [7]-[10], which measure radial displacement of the skin surface, the AlN thin film sensor is used to measure changes in circumferential length of the skin surface, and the axis of the strain is set as the length direction of the sensor. With this configuration, the thin thickness of this sensor leads to high sensitivity.

This paper is organized as follows: Section II introduces 


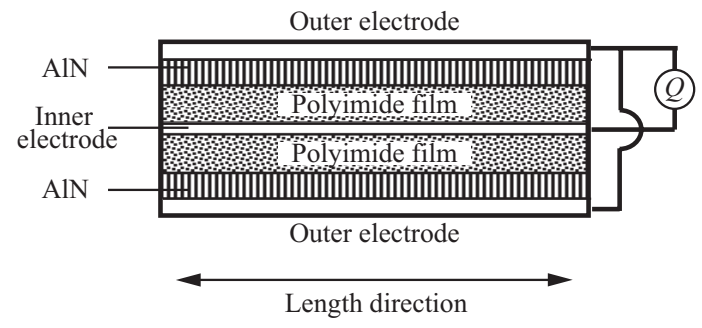

Fig. 2. Laminated structure of the AlN thin film sensor. The strain of sensor is in the length direction.

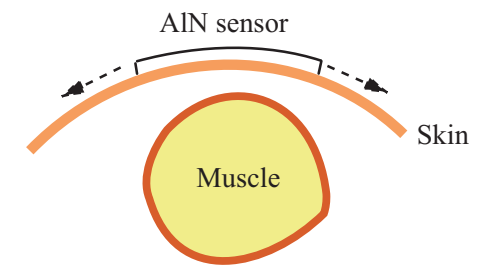

Fig. 3. A schematic view of the signal acquisition using an AlN thin film sensor. When the cross sectional area of the muscle increases, the sensor is stretched in the length direction.

the AlN thin film sensor and explains details of the proposed signal acquisition method. Then, a motion classification method is developed based on a probabilistic neural network (NN). In Section III, performance of the proposed method is verified with experiments. Finally, Section IV concludes this paper.

\section{METHOD}

\section{A. AlN Thin Film Sensor}

Aluminum Nitride (AlN) is one of piezoelectric ceramic materials. Because of the piezoelectric properties of AlN, epitaxially grown crystalline AIN has been deposited mostly on silicon wafers or sapphire substrates. Our previous researches have reported that highly $c$-axis oriented AlN thin film can also be deposited on various substrates, like aluminum foil, polyethylene terephthalate (PET), and polyimide films [12]-[14]. Since AlN keeps piezoelectric characteristics at temperature up to $1150{ }^{\circ} \mathrm{C}$, this sensor shows excellent thermal and chemical stability, and these properties facilitate a variety of practical applications.

A laminated structure of the AlN thin film sensor is shown in Fig. 2. This sensor consists of three Pt electrode layers, two AlN layers and two polyimide layers, and the total thickness is less than $40 \mu \mathrm{m}$. Since the inner electrode is shielded by the outer electrodes, this sensor is more robust to noise. A charge amplifier is used to convert the sensor's output into voltage signals.

\section{B. Signal Acquisition using an AlN Film Sensor}

Fig. 3 shows a schematic view of the proposed signal acquisition method with an AlN film sensor attached on the skin surface. During muscle's contraction, the AlN sensor is stretched due to the increase of the cross-sectional muscle area. The strain $\epsilon(t)$ in the AlN film can be measured, and a

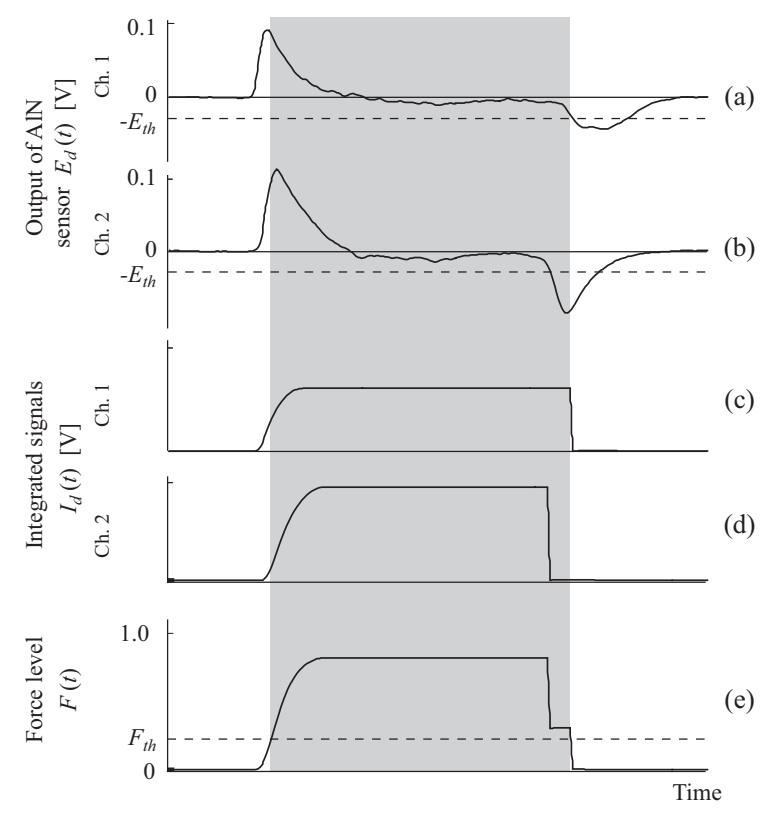

Fig. 4. An example of the signals in the proposed feature extraction process.

voltage output $E(t)$ is obtained via a charge amplifier. The output of AlN sensor is proportional to the mechanical strain. Like all piezoelectric materials, however, AlN sensor is not suitable for static measurement. A decay of the output signal can be found when the strain keeps constant. Fig. 4. (a) and (b) depict examples of the signals of AlN sensors. It can be found that the first increase of $E(t)$ corresponds to a muscle contraction. When the muscle is relaxed, the output voltage is of opposite polarity.

Suppose there are $D$ channels of AlN sensor signals, denoted as $E_{d}(t)(d=1,2, \cdots, D)$. A threshold $E_{t h}$ is applied to the signals to reduce noise due to subject's subtle involuntary movements and noise induced by the signal conditioning circuits. The modified signal $E_{d}^{\prime}(t)$ is defined as follows,

$$
E_{d}^{\prime}(t)= \begin{cases}E_{d}(t)-E_{t h} & \left(E_{d}(t)>E_{t h}\right) \\ 0 & \left(\left|E_{d}(t)\right|<E_{t h}\right) \\ E_{d}(t)+E_{t h} & \left(E_{d}(t)<-E_{t h}\right)\end{cases}
$$

The signal $E_{d}^{\prime}(t)$ is integrated using the trapezoid rule. The resulted signal $I_{d}(t)$ shows similar trend to the increase of cross-sectional muscle area (see Fig. 4 (c) and (d)). When $E_{d}(t)<-E_{t h}, I_{d}(t)$ is reset to zero.

Then, a ratio $\alpha_{d}(t)$ is defined as

$$
\alpha_{d}(t)=\frac{I_{d}(t)}{I_{d}^{\max }}
$$

where $I_{d}^{\max }$ is the value of $I_{d}(t)$ measured in a maximum voluntary contraction. The ratio $\alpha_{d}(t)$ is further normalized to make the sum of $D$ channels equal to 1 ,

$$
x_{d}(t)=\frac{\alpha_{d}(t)}{\sum_{d^{\prime}=1}^{D} \alpha_{d^{\prime}}(t)}
$$

The feature vector $\mathbf{x}(t)=\left[x_{1}(t), x_{2}(t), \cdots, x_{D}(t)\right]$ is used as input to motion classification process. 


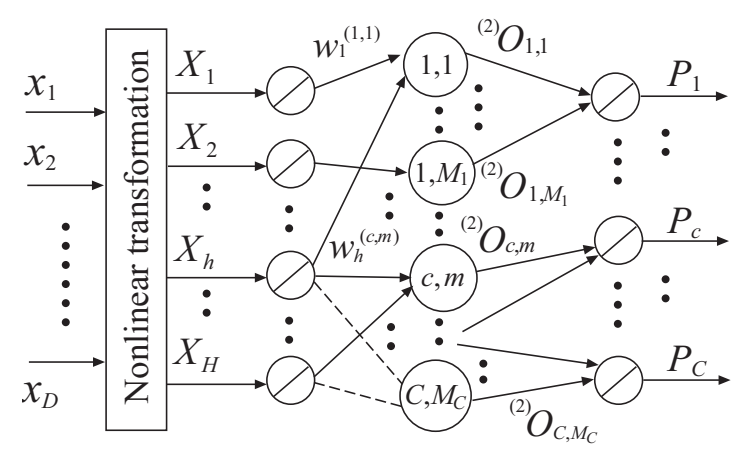

Fig. 5. The structure of LLGMN [15].

\section{Motion Classification using a Probabilistic NN}

In the proposed method, a probabilistic NN, called loglinearized Gaussian mixture network (LLGMN) [15], is used for motion classification. LLGMN is based on the Gaussian mixture model (GMM) and the log-linear model of probability distribution function (pdf). By applying the log-linear model to a product of the mixture coefficients and the mixture components of GMM, this semiparametric model of pdfs is incorporated into a three-layer feedforward $\mathrm{NN}$ as shown in Fig. 5. LLGMN estimates probability distribution of motions based on the input feature vector $\mathbf{x}(t)$, and outputs posterior probability of motion $c(c=1, \cdots, C)$. For details of LLGMN, please refer to [15].

It is assumed that amplitude of the force during motions changes in proportion to $I_{d}(t)$. A force level $F(t)$ is defined as

$$
F(t)=\frac{1}{D} \sum_{d=1}^{D} \alpha_{d}(t) .
$$

In order to recognize whether the motion has really occurred or not, the force level $F(t)$ is compared with a predefined motion appearance threshold $F_{t h}$. A motion is considered to have occurred if $F(t)$ exceeds $F_{t h}$. The gray area in Fig. 4 indicates the duration of motion.

In addition, the entropy of LLGMN's outputs is calculated to present the risk of misclassification. The entropy is defined as

$$
H(t)=-\sum_{c=1}^{C} P_{c}(t) \log P_{c}(t)
$$

where $P_{c}(t)$ stands for the posterior probability of motion $c$. If the entropy $H(t)$ is less than a threshold $H_{d}$, the specific motion with the largest probability is determined according to the Bayes' decision rule. Otherwise, the determination is suspended. Furthermore, a motion decision threshold $O_{t h}$ is adopted in this study. A motion is considered to have occurred only if this motion has been classified continuously more than $O_{t h}$ times.

\section{EXPERIMENTAL RESULTS}

\section{A. Strain Measurement using AlN Sensor}

In order to investigate response characteristics of the AlN sensor in the length direction, cantilever beam experiments

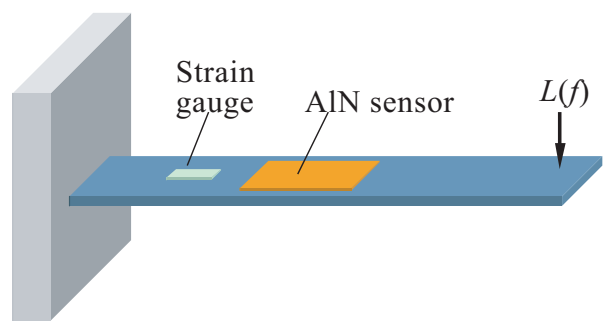

Fig. 6. Setup of the cantilever beam experiments.
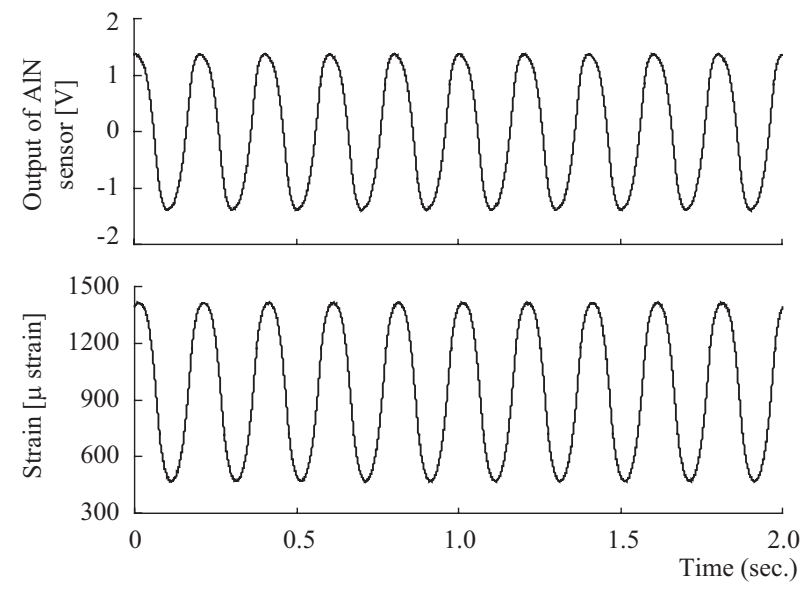

Fig. 7. Examples of signals measured with the AlN sensor (top) and the strain gauge (bottom) for $f=5 \mathrm{~Hz}$.

were conducted. The experimental setup is shown in Fig. 6 . A thin stainless steel board $(45 \mathrm{~mm} \times 10 \mathrm{~mm} \times 0.3 \mathrm{~mm})$ was used as the cantilever beam. A sinusoidal load $L(f)$ was applied at the free end, where $f$ is the frequency of the load. An AlN sensor of $10 \mathrm{~mm}$ length and $8 \mathrm{~mm}$ width was attached at the middle part of the beam using epoxy glue, and a strain gauge was installed to measure the strain of the beam.

Signals of the AlN sensor were input into a charge amplifier (Model-4001B, Showa Sokki Corp.). The sensitivity of the charge amplifier was set as $50 \mathrm{pC} / \mathrm{V}$. A strain amplifier (DPM-713B, Kyowa Electronic Instruments) is used for signal conditioning for the strain gauge. Then, the signals from both the AlN sensor and the strain gauge were acquired with a 14-bit A/D converter (USB-6009, National Instruments), and the sampling frequency was $2 \mathrm{kHz}$ in these experiments.

Fig. 7 shows examples of the signals measured with the AlN sensor and the strain gauge for $f=5 \mathrm{~Hz}$. It can be observed that output of the AlN sensor agrees well with the strain of the beam. Moreover, frequency response of the AlN sensor was studied for frequencies in the range of $[0.1,130] \mathrm{Hz}$, and the gain of AlN signal to strain was obtained. The linearity in output for increasing strain, for frequencies of $0.1,1,5$, and $10 \mathrm{~Hz}$, is shown in Fig. 8. For all four frequencies, high linearity of the AlN sensor can be confirmed. Except at $f=0.1 \mathrm{~Hz}$, the gain of AlN signal to strain is about $3 \mathrm{mV} / \mu$ strain. For the case of $f=0.1 \mathrm{~Hz}$, the gain decreases by half. It is considered that the decrease 


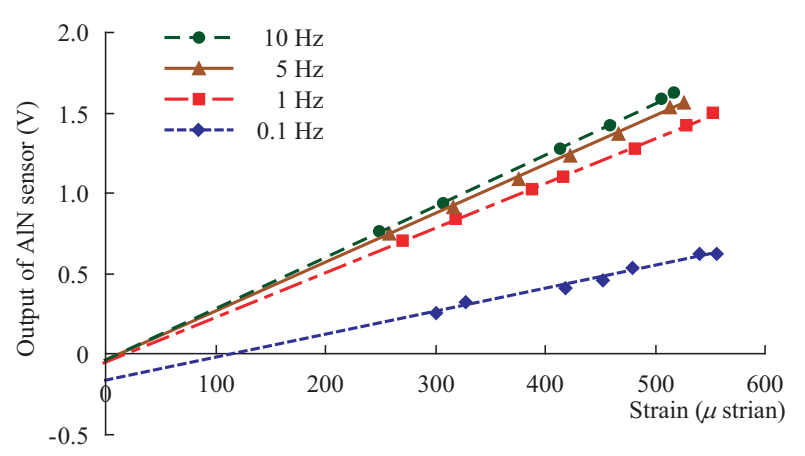

Fig. 8. Linearity of the AlN sensor.

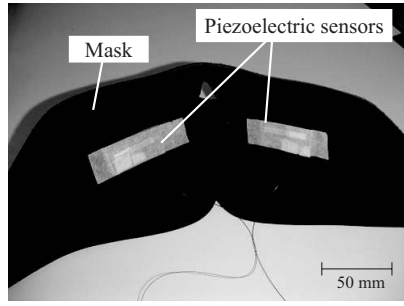

(a)

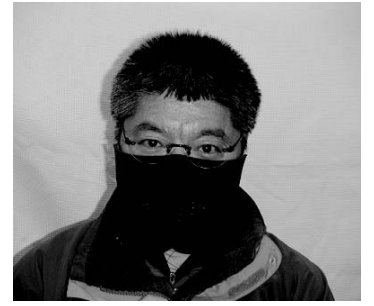

(b)
Fig. 9. The mask used in the experiments. (a) The mask with two AIN sensors installed. (b) A subject wearing the mask.

is mostly due to the response characteristics of the charge amplifier.

\section{B. Motion Classification Experiments}

Motion classification experiments were conducted to examine performance of the proposed method. Five subjects (A, B, C, and D: healthy, E: a patient with cervical spine injury) participated in the experiments.

Two AlN sensors were attached to a mask using singlecoated tape to measure the movements of facial muscles at the right and the left sides of mouth (Fig. 9). Dimension of the AlN sensors was $30 \mathrm{~mm} \times 5 \mathrm{~mm}$. Two sets of simplified charge amplifiers (T-CAM001B: TURTLE Industry) were used. In these experiments, three motions were classified ( $C=3$ : right side contraction, left side contraction, contraction at both sides). Each subject achieved ten trials of these motions. In each trail, the subjects were asked to achieve motions in the order, and each motion was kept for three seconds with seven seconds rest between two motions. The sampling frequency was $1 \mathrm{kHz}$ in these experiments. LLGMN was trained previously for each subject. Parameters in the feature extraction and motion classification processes were set as: $E_{t h}=0.025, F_{t h}=0.25, O_{t h}=100$, and $H_{d}$ was set as 0.2 for subjects $\mathrm{A}$ and $\mathrm{E}, 0.3$ for the others.

An example of the classification result of subject $B$ is shown in Fig. 10. In this figure, two channels of the output of AlN sensors, two channels of the integrated signals, the force level $F(t)$, and the classification results are plotted. The gray areas indicate that motion was determined because the force level exceeds the threshold $F_{t h}$. It can be seen that the proposed method achieves high classification performance.

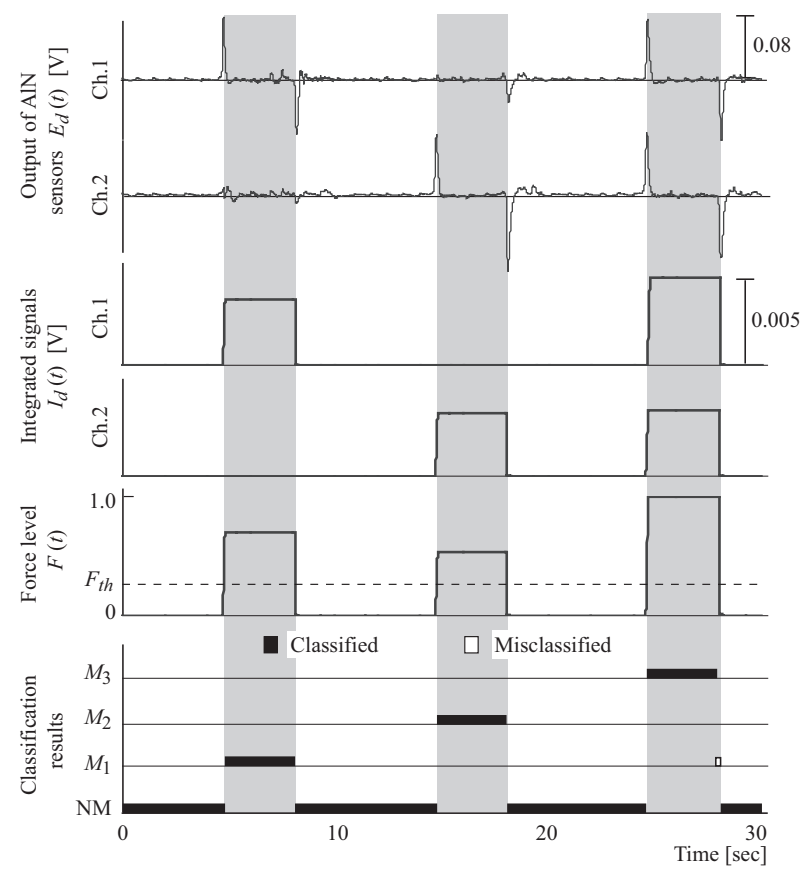

Fig. 10. An example of the classification result (Subject B). ( $M_{1}$ : right side contraction; $M_{2}$ : left side contraction; $M_{3}$ : contraction at both sides; NM: No motion)

TABLE I

ClassifiCATION RATES FOR FIVE SUBJECTS (\%).

\begin{tabular}{l|l|l}
\hline & Mean & S.D. \\
\hline Subject A & 99.93 & 0.12 \\
Subject B & 99.74 & 0.45 \\
Subject C & 99.87 & 0.22 \\
Subject D & 99.65 & 0.36 \\
Subject E & 99.88 & 0.20 \\
\hline
\end{tabular}

Misclassification is found at the end of motion 3. It is considered that the misclassification is caused by the difference of the timings, at which the integrated signals are reset to zero. Also, such misclassification could be reduced using an appropriately modulated threshold $O_{t h}$.

Accuracy of the classification results for five subjects was investigated as well. Table I depicts the mean values and standard deviations (S.D.) of the classification rates, for ten independent trials. All five subjects achieved a classification rate more than $99 \%$.

\section{CONCLUSION}

In this study, a novel method was proposed for measurement of muscle movements for human interfaces. The proposed method utilizes a flexible AlN piezoelectric thin film sensor for signal acquisition. This sensor measures morphological changes of muscles at the skin surface. Then a motion classification method has been developed with a probabilistic NN, i.e. LLGMN. Results in the evaluation experiments suggest that the AlN sensor can measure strain applied in the length direction successfully. Finally, high classification rates have been confirmed in the motion classification experiments. Control signals for human interfaces 
can be generated with the motions classified.

As shown in Fig. 8, we can find significant difference between frequencies $f \geq 1 \mathrm{~Hz}$ and those $f<1 \mathrm{~Hz}$. With respect to the fact that most of muscle movements are slow (less than $1 \mathrm{~Hz}$ ), dedicated design of charge amplifier, especially for low frequencies, is required. In the future research, additional experiments are needed to evaluate the proposed method over other subjects, and comparison experiments, between the proposed method and traditional methods like human interfaces based on EMG or MMG signals, is helpful to improve the proposed method. We would like to enhance the feature extraction algorithm used in the proposed method. Also, human interface systems would be developed with the proposed method.

\section{ACKNOWLEDGMENTS}

The authors gratefully acknowledge participation of the volunteers in this study. This work was partially supported by KAKENHI (20700472).

\section{REFERENCES}

[1] M. F. Kelly, P. Parker, and R. N. Scott, "The application of neural networks to myoelectric signal analysis: A preliminary study", IEEE Trans. Biomed. Eng., vol. 37, 1990, pp. 221-230.

[2] T. Tsuji, O. Fukuda, M. Kaneko, and K. Ito, "Pattern classification of time-series EMG signals using neural networks", Int. J. Adapt. Contr. Signal Process., vol. 14, 2000, pp. 829-848.

[3] K. Englehart and B. Hudgins, "A robust, real-time control scheme for multifunction myoelectric control", IEEE Trans. Biomed. Eng., vol. 50, 2003, pp. 848-854.

[4] D. Graupe, J. Magnussen, and A. A. M. Beex, "A microprocessor system for multifunctional control of upper limb prostheses via myoelectric signal identification", IEEE Trans. Automat. Contr., vol. 23, 1978, pp. 538-544.
[5] K.R. Wheeler and C.C. Jorgensen, "Gestures as input: Neuroelectric joysticks and keyboards", IEEE Pervasive Computing, vol. 2, no. 2, 2003, pp. 56-61.

[6] O. Fukuda, T. Tsuji, M. Kaneko, and A. Ohtsuka, "A human-assisting manipulator teleoperated by EMG signals and arm motions", IEEE Trans. Robot. Autom., vol. 19, 2003, pp. 210-222.

[7] L.P.J. Kenney, I. Lisitsa, P. Bowker, G.H. Heath, and D. Howard, "Dimensional change in muscle as a control signal for powered upper limb prostheses: a pilot study", Med. Eng. Phys., vol. 21, 1999, pp. 589-597.

[8] T. Žagar and D. Križaj, "Validation of an accelerometer for determination of muscle belly radial displacement", Med. Biol. Eng. Comput., vol. 43, 2005, pp. 78-84.

[9] J. Silva, W. Heim, and T. Chau, "MMG-based classification of muscle activity for prosthesis control", Proc. of 26th Annual Inter. Conf. of the IEEE Engineering in Medicine and Biology Society, 2004, pp. 968971

[10] T. Tsuji, T. Yoshihisa, and K. Shima, "An MMG-based control method of prosthetic manipulators using acceleration sensors", Journal of the Robotics Society of Japan, vol. 25, 2007, pp. 874-880. (in Japanese)

[11] Y.P. Zheng, M.M.F. Chan, J. Shi, X. Chen, and Q.H. Huang, "Sonomyography: Monitoring morphological changes of forearm muscles in actions with the feasibility for the control of powered prosthesis", Med. Eng. Phys., vol. 28, 2006, pp. 405-415.

[12] N. Ueno, M. Akiyama, K. Ikeda, and H. Takeyama, "A foil type pressure sensor using nitelide aluminum thin film", Trans. of SICE, vol. 38, 2002, pp. 427-432. (in Japanese)

[13] M. Akiyama et al., "Flexible piezoelectric pressure sensors using oriented aluminum nitride thin films prepared on polyethylene terephthalate films", J. Appl. Phys., vol. 100, 2006, Article No. 114318.

[14] $\mathrm{N}$. Bu, N. Ueno, and O. Fukuda, "Monitoring of respiration and heartbeat during sleep using a flexible piezoelectric film sensor and empirical mode decomposition", Proc. of 29th Annual Inter. Conf. of the IEEE Engineering in Medicine and Biology Society, 2007, pp. 1362-1366.

[15] T. Tsuji, O. Fukuda, H. Ichinobu, and M. Kaneko, "A log-linearized Gaussian mixture network and its application to EEG pattern classification", IEEE Trans. Syst. Man Cybern. C Appl. Rev., vol. 29, 1999, pp. $60-72$ 